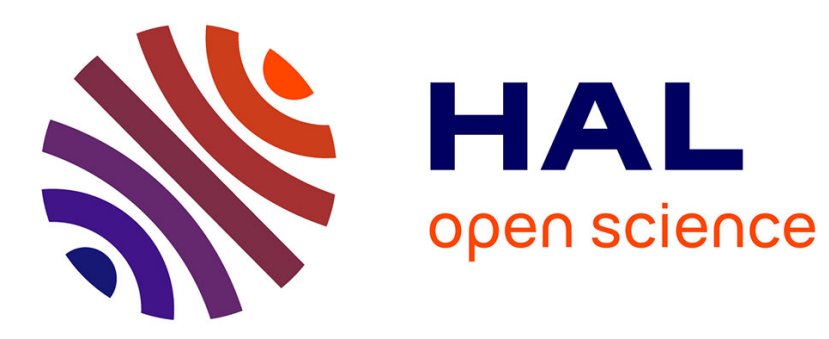

\title{
Smooth transition of AUV motion control: From fully-actuated to under-actuated configuration
}

\author{
Xianbo Xiang, Lionel Lapierre, Bruno Jouvencel
}

\section{To cite this version:}

Xianbo Xiang, Lionel Lapierre, Bruno Jouvencel. Smooth transition of AUV motion control: From fully-actuated to under-actuated configuration. Robotics and Autonomous Systems, 2015, Advances in Autonomous Underwater Robotics, 67, pp.14-22. 10.1016/j.robot.2014.09.024 lirmm-01310976

\section{HAL Id: lirmm-01310976 https://hal-lirmm.ccsd.cnrs.fr/lirmm-01310976}

Submitted on 3 May 2016

HAL is a multi-disciplinary open access archive for the deposit and dissemination of scientific research documents, whether they are published or not. The documents may come from teaching and research institutions in France or abroad, or from public or private research centers.
L'archive ouverte pluridisciplinaire HAL, est destinée au dépôt et à la diffusion de documents scientifiques de niveau recherche, publiés ou non, émanant des établissements d'enseignement et de recherche français ou étrangers, des laboratoires publics ou privés. 


\title{
Smooth transition of AUV motion control: From fully-actuated to under-actuated configuration
}

\author{
Xianbo Xiang ${ }^{\mathrm{a}, *}$, Lionel Lapierre ${ }^{\mathrm{b}}$, Bruno Jouvencel ${ }^{\mathrm{b}}$ \\ a School of Naval Architecture and Ocean Engineering, Huazhong University of Science and Technology, 1037, Luoyu Road, 430074, Wuhan, China \\ ${ }^{\mathrm{b}}$ Department of Robotics, CNRS-LIRMM-UMII, UMR 5506-CC 477, 161 rue Ada, 34392 Montpellier Cedex 5, France
}

\section{H I G H L I G H T S}

- Steering AUV through the whole low-speed and high-speed profiles is considered.

- Evolution of the side-slip angle is clearly treated in the motion control design.

- Smooth control transition between fully/under-actuated configurations is enabled.

- Simulation results explicitly show the transition behaviors of the control efforts.

\section{A R T I C L E I N F O}

\section{Article history:}

Available online 8 October 2014

\section{Keywords:}

AUV

Path following

Side-slip angle

Underactuation

\begin{abstract}
A B S T R A C T
This paper addresses the problem of steering autonomous underwater vehicle (AUV) along a desired horizontal path throughout the full-range low-speed and high-speed profiles, experiencing both fullyactuated and under-actuated configurations. First, a nonlinear controller adopting Lyapunov's direct method and backstepping technique is proposed for under-actuated AUV, based on the Line-of-Sight guidance built in a moving Frenet-Serret frame. And then, the controller is adapted to fully-actuated AUV except that the control computation for the evolution of the side-slip angle is different from the case of under-actuated one. Hence, both the fully-actuated and under-actuated configurations are under the same control framework, which enables a smooth continuous transition between two configurations in a synthesized controller. Finally, simulation results illustrate the performance of the proposed control design, where the varied control efforts in the sway direction clearly show the transitions from fullyactuated to under-actuated configuration.
\end{abstract}

(C) 2014 Elsevier B.V. All rights reserved.

\section{Introduction}

Over the past two decades, a remarkable growth regarding the operation of autonomous underwater vehicle (AUV) has been witnessed in the wide range of commercial, scientific and military applications [1-4], such as offshore oil and gas exploration and exploitation, underwater survey and observation, mine reconnaissance and neutralization, etc. In order to meet these miscellaneous goals, it is desirable to automatically control the AUV through all the feasible speed profiles from low-speed starting to high-speed maneuvering. Traditionally, fully actuated AUV with independent actuators in all degrees of freedom (DOF) simultaneously are

\footnotetext{
* Corresponding author. Tel.: +86 27 87543157; fax: +86 2787542146 .

E-mail addresses: xbxiang@hust.edu.cn, xiangxb@gmail.com (X. Xiang), lapierre@lirmm.fr (L. Lapierre), jouvencel@lirmm.fr (B. Jouvencel).
}

suitable for low-speed maneuvering in confined water and easily docking in harbor, whereas under-actuated AUV possessing more DOF than the control inputs, which are not able to command independent accelerations in all DOF simultaneously, are assumed for high-speed maneuvering in long-range and long-duration missions due to cost-effective and weight considerations. In practice, lots of AUVs are inherently under-actuated without thrusters and/or rudders in the sway, heave or roll directions, as described in [5-9], to name but a few. In addition, a fully-actuated AUV equipped with lateral actuators in sway and heave directions to assist at low-speed maneuvering, dramatically decreases its efficiency in these lateral directions at high-speed forward movement due to the relative perpendicular water flow passing the outlets, which implies that the sway and heave movements are not independently controlled, and leads a fully-actuated AUV to behave like an under-actuated one as the rest. It results in the development of structurally different controllers for both the fullyactuated and under-actuated configurations, and an intelligent 
supervisor is generally required to perform a heuristics and hybrid switch between two controllers. On the other hand, fully actuated underwater vehicles might be exposed to actuator failures, rendering themselves into under-actuated cases. Hence, it is also required for a critical solution to have control redundancy to guarantee the vehicle safety as much as possible, by allowing the vehicle to be controlled with the remained actuator capability, and enabling the control algorithm to switch between fully-actuated and under-actuated configurations to ensure system reliability. Moreover, from both a theoretical and practical point of view, it is desirable to have a smooth transition between these two actuated configurations covering the full-range speed profile in a single synthesized controller, in order to avoid the possible oscillation and even destabilization problem coming from hard switching, reduce the complexity of the controller and render easy implementation in practice.

To the best knowledge of the authors, there are few research work reported in this specific control topic to deal with the underactuated and fully-actuated AUV configurations together. In [10], a hybrid switching design combining a dynamic positioning controller in low-speed and a track-keeping controller in high-speed is proposed for minehunters. In [11], an automatic navigation and track-keeping system (ANTS) dealing with tight heading control are separated from a harbor mode with high-precision position and heading control. In [12], a simple logic is used to switch algorithms when the operation changes. Reinitialization of control parameter is required and discontinuity occurs at the point of switching operation. In [13], simultaneously global asymptotic stabilization and tracking is only solved in the case of under-actuated underwater vehicle, without the consideration from fully-actuated to under-actuated configurations. In [14], a unified control structure for AUV is proposed where the transition factor relies on the composite speed; however, the derivative evolution of side-slip angle is not thoroughly analyzed in the dynamics stage, which implies different control treatments due to the directly or indirectly controlled transverse (sway) movement in fully-actuated and under-actuated modes respectively. On the other hand, while developing advanced methods for AUV motion control, it should be noticed that kinematic and dynamic models of AUVs are highly nonlinear and coupled [15], making the motion control design a challenging task. The complex hydrodynamics effects, which must be taken into account during the control design, excludes any attempt to design a steering system for the AUV relying on its kinematic model only as stated in [7]. In addition, underactuation rules out the use of trivial control schemes, e.g., full state-feedback linearization [16]. Furthermore, the indirectly controlled sway and heave velocities due to underactuations, which generate non-zero angles of side-slip and attack respectively, should be carefully considered as well [17].

Motivated by the above considerations, this paper proposes a synthesized path following controller which enables smooth transition between fully-actuated and under-actuated AUV configurations throughout the full-range feasible speed profile. For sake of simplicity, 3-DOF horizontal motion control of AUVs is considered herein, and it can be extended to 6-DOF motion control in full space if the heave speed in the decoupled vertical plane and resulted attack angle are included, besides the side-slip angle in the sway direction. The control system proposed in this paper is derived via two steps. First, by adopting Lyapunov's direct method and backstepping technique, a nonlinear path following control law for fully-actuated AUV is proposed based on Line-of-Sight guidance built-in Frenet-Serret frame, which is adapted from the control law for under-actuated AUV originally proposed in [18]. Thus, both the under-actuated and fully-actuated cases under the same control framework, except that the control computation for the derivatives of the side-slip angle of AUV is different, through completely considering the difference of the indirectly or directly controlled sideslip angle in these two cases. And then, a smooth transition but not hard switch between two controllers is designed from low-speed starting in fully-actuated pattern to high-speed maneuvering in under-actuated pattern, where the transition factor covering the full-range speed profile is a smooth function of the instantaneous surge speed of the AUV. Consequently, the desired control design is completed for AUV traveling from low speed (fully-actuated configuration) to high speed (under-actuated configuration).

The rest of the paper is organized as follows. Problem statement is presented in the next section, including the kinematics and dynamics model of AUV and the control objective. In Section 3, a nonlinear path following controller is designed for under-actuated AUV, and then extended to fully-actuated AUV in order to keep the same control framework for both actuation configurations. Subsequently, a synthesized controller with smooth continuous transition in terms of the instantaneous surge speed of AUV is proposed. Numerical simulation results are given in Section 4 to illustrate the performance of the proposed controller. Section 5 contains some concluding remarks and discusses problems that warrant further research.

\section{Problem statement}

This section describes the kinematic and dynamic model of the AUV in the horizontal plane and formulates the motion control problem of the path following through the full-range feasible speed profiles. The notation used in the paper is standard [19].

\subsection{AUV modeling}

Following standard practice, the general kinematic and dynamic mode of the AUV in the horizontal plane can be described by the motion components in surge, sway and yaw directions. The kinematic equations take the form

$\left\{\begin{array}{l}\dot{x}=u \cos \psi-v \sin \psi \\ \dot{y}=u \sin \psi+v \cos \psi \\ \dot{\psi}=r\end{array}\right.$

where $x$ and $y$ are the coordinates of its center of mass expressed in the inertial frame, and $\psi$ defines its orientation (heading angle). Surge speed $u$, sway speed $v$ and yaw speed $r$ denote the AUV body-fixed linear and angular velocities with respect to the inertial frame.

Neglecting the motions in heave, roll and pitch, the 3-DOF dynamic equations of the AUV in the horizontal plane is simplified as [20]

$\left\{\begin{array}{l}\tau_{u}=m_{u} \dot{u}-m_{v} v r+d_{u} u \\ \tau_{v}=m_{v} \dot{v}+m_{u} u r+d_{v} v \\ \tau_{r}=m_{r} \dot{r}-m_{u v} u v+d_{r} r\end{array}\right.$

with

$m_{u}=m-X_{\dot{u}} \quad d_{u}=-X_{u}-X_{|u| u}|u|$

$m_{v}=m-Y_{\dot{v}} \quad d_{v}=-Y_{v}-Y_{|v| v}|v|$

$m_{r}=I_{z}-N_{\dot{r}} \quad d_{r}=-N_{r}-N_{|r| r}|r|$

$m_{u v}=m_{u}-m_{v}$

where $m$ denotes the AUV mass, $X_{(\cdot)}, Y_{(\cdot)}, N_{(\cdot)}$ express hydrodynamic derivatives of the system, and $d_{(\cdot)}$ capture hydrodynamic damping effects.

Eq. (2) can be taken as a unified dynamics model for both the under-actuated and fully-actuated AUV configurations. The differences of the dynamic equations between fully-actuated and under-actuated AUVs are as follows: 


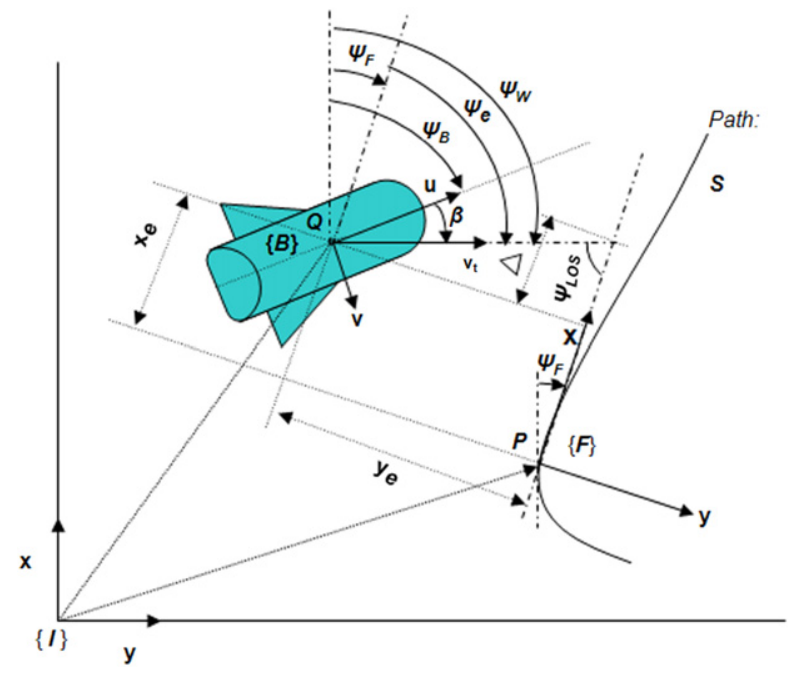

Fig. 1. Frame definitions of AUV path following.

(1) In the case of fully actuation, $\tau_{u}, \tau_{v}$ denote the external forces acting on the AUV in surge and sway directions, respectively. $\tau_{r}$ denotes the external torque about the $z$-axis of the AUV.

(2) In the case of high-speed traveling, dramatically dropped efficiency of lateral thruster in the sway direction results in the degeneration of fully-actuated AUV configuration into underactuated one, such that the force $\tau_{v}=0$ in the under-actuated AUV dynamics equation.

\subsection{Problem formulation}

Generally, there are two main types of motion control problem covering the full-range nonzero speed profile, namely, trajectory tracking and path following. Since smoother convergence to a path is achieved in the path following instead of trajectory tracking control laws and the control signals are less likely pushed to saturation $[18,21,22]$, only path following strategy is considered in this paper.

As depicted in Fig. 1, an under/fully-actuated AUV follows a predefined planar path $S, P$ is a moving point (acting as a virtual target) on the path to be followed, and $Q$ is the center of mass of the AUV. Associated with $P$, the corresponding Frenet-Serret frame $\{F\}$ can be built [23]. The path $S$ is parameterized by the moving target $P$ with curvilinear abscissa (along the path length) denoted by $s$.

Let $\left(x_{e}, y_{e}\right)$ denote the coordinates of $Q$ in $\{F\}$, where the alongtrack error $x_{e}$ represents the distance from vehicle to the desired position of the virtual target along the $x$-axis of $\{F\}$, and the crosstrack error $y_{e}$ represents the distance along the $y$-axis of $\{F\}$. Let the rotations from $\{I\}$ to $\{F\}$ and from $\{I\}$ to $\{B\}$ be denoted by the angles $\psi_{F}$ and $\psi_{B}$, respectively, and let $c_{c}(s)$ denote the path curvature. The side-slip angle is defined as $\beta=\arctan (v / u)$, and the composite speed is $v_{t}=\sqrt{u^{2}+v^{2}}$.

The problem of path-following control for an under/fullyactuated AUV can be formulated as follows:

Given a planar path, develop feedback control laws for external forces and torque acting on an under/fully-actuated AUV, such that its center of mass asymptotically converges to the path, while its surge speed tracks a desired profile and the composite speed aligns with the tangent vector of the path.

\section{Path following control design}

\subsection{Path following error dynamics}

Let the position and course angle of the AUV denoted by $Q=$ $\left(x, y, \psi_{W}\right)^{T}$ in the inertial frame $\{I\}$ as illustrated in Fig. 1, and let the position and heading of the moving virtual target on the path denoted by $P=\left(x_{F}, y_{F}, \psi_{F}\right)^{T}$ in the inertial frame $\{I\}$. The path following error vector $p_{e F}=\left(x_{e}, y_{e}, \psi_{e}\right)^{T}$ built in the Frenet-Serret frame $\{F\}$ can be written as

$$
\left[\begin{array}{l}
x_{e} \\
y_{e} \\
\psi_{e}
\end{array}\right]=\left[\begin{array}{ccc}
\cos \psi_{F} & \sin \psi_{F} & 0 \\
-\sin \psi_{F} & \cos \psi_{F} & 0 \\
0 & 0 & 1
\end{array}\right]\left[\begin{array}{c}
x-x_{F} \\
y-y_{F} \\
\psi_{W}-\psi_{F}
\end{array}\right]
$$

where the course angle $\psi_{W}=\psi_{B}+\beta$ and yaw rate $r=\dot{\psi}_{B}$.

Differentiating the error vector (4) and recalling $\dot{\psi}_{F}=c_{c}(s) \dot{s}$ yields the error dynamics built in the Frenet-Serret frame $\{F\}$

$$
\left[\begin{array}{c}
\dot{x}_{e} \\
\dot{y}_{e} \\
\dot{\psi}_{e}
\end{array}\right]=\left[\begin{array}{c}
-\dot{s}\left(1-c_{c} y_{e}\right)+v_{t} \cos \psi_{e} \\
-c_{c} \dot{s} x_{e}+v_{t} \sin \psi_{e} \\
r+\dot{\beta}-c_{c} \dot{s}
\end{array}\right] .
$$

\subsection{LOS heading guidance}

Line-of-Sight (LOS) is a popular heading guidance applied in the marine vehicle system. Traditionally, LOS guidance is built in the inertial frame to help vehicles follow a straight-line path constructed by way points $[24,25]$. In this paper, LOS guidance is adapted and built in a moving Frenet-Serret frame, for AUVs following any feasible curved path.

As depicted in Fig. 2(a), the coordinate origin of the AUV is $(x, y)$, and the LOS shooting point on the straight-line path is $\left(x_{l o s}, y_{l o s}\right)$. Thus, the desired yaw angle under LOS guidance in the inertial frame is $\psi_{\text {los }}=\arctan \left(\frac{y_{\text {los }}-y}{\Delta}\right)$ where the look ahead distance $\Delta$ is constant. The choice of $\Delta$ is instrumental to shape the vessel moving towards the straight-line path [24].

In this paper, the LOS heading guidance is built in a moving Frenet-Serret frame $\{F\}$ along the evolution of the virtual target

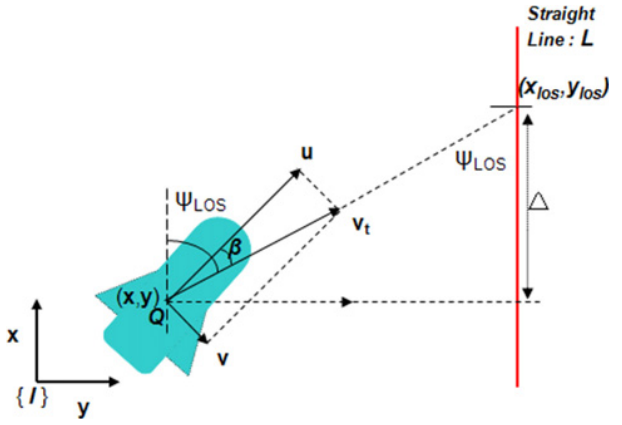

(a) LOS built in Inertial frame.

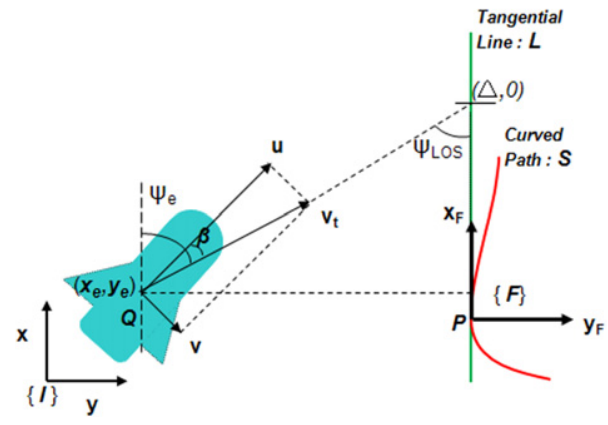

(b) LOS built in Frenet-Serret frame.

Fig. 2. LOS guidance for straight-line and curved paths in different frames. 
$P$ on the path, when the AUV tracks arbitrary feasible curved path illustrated in Fig. 2(b). The traditional parameter $\Delta$ is extended to look at the distance along the tangential path in $\{F\}$. When the path has a small radius of the tangent osculating circle (i.e., large curvature in the Frenet-Serret frame) at one reference point $P$, it is better to increase the heading guidance to adhere it to the sharp turning of the path at that moment. On the contrary, the heading guidance is decreased when the path is smooth. In this sense, $\Delta$ can be chosen as a variable function of the curvature $c_{c}(s)$.

Revisiting Figs 1 and 2(b), $\psi_{e}$ is equal to the desired LOS guidance $\psi_{\text {los }}$ in the case of perfect path following control. It means the LOS guidance in the Frenet-Serret frame can be defined as

$\psi_{\text {los }}=-\arcsin \left(\frac{y_{e}}{\sqrt{y_{e}^{2}+\Delta^{2}}}\right)$

with $\Delta=2 L-\operatorname{Lsat}\left(k_{0}\left|c_{c}(s)\right|\right)$, where $0<k_{0}<k_{0 \max }, L$ is the longitudinal length of the vehicle, and sat $(\cdot)$ is the saturation function in $(-1,1)$. Thus, a large $\Delta$ brings a mild approaching guidance to a smooth curved path, while a small $\Delta$ brings a aggressive approach to a sharp path. In the case of straight-line path where $c_{c}(s)=0, \Delta$ is equal to two vehicle's length, which is corresponding to a standard choice in LOS guidance.

Moreover, as we can see later, the adapted LOS guidance is also instrumental in path following controller design to improve the convergence with ULES or UGES performance.

\subsection{Path following control design for under-actuated AUV}

The path following control design is first conducted for underactuated AUV, which is more complicated than that of fullyactuated AUV due to the indirectly controlled sway movement.

\subsubsection{Kinematic controller}

As the main objective of the path following control is to drive the error vector $\left(x_{e}, y_{e}, \psi_{e}\right)^{T}$ to zero, and the LOS guidance is used to decrease the course error, the following candidate Lyapunov function can be considered

$V_{1}=\frac{1}{2}\left[x_{e}^{2}+y_{e}^{2}+\left(\psi_{e}-\psi_{\text {los }}\right)^{2}\right]$.

Resorting to the error dynamics model in (5), the derivative of $V_{1}$ is

$$
\begin{aligned}
\dot{V}_{1}= & -x_{e} \dot{s}+v_{t} s_{1} \cos \psi_{e}+v_{t} y_{e} \sin \psi \\
& +\left(\psi_{e}-\psi_{l o s}\right)\left(\dot{\psi}_{e}-\dot{\psi}_{l o s}\right) .
\end{aligned}
$$

It is straightforward to choose the kinematic control laws as

$$
\left\{\begin{array}{l}
\dot{s}=k_{1} x_{e}+v_{t} \cos \psi_{e} \\
\dot{\psi}_{e}=\dot{\psi}_{l o s}-y_{e} v_{t} \frac{\sin \psi_{e}-\sin \psi_{l o s}}{\psi_{e}-\psi_{l o s}}-k_{2}\left(\psi_{e}-\psi_{l o s}\right)
\end{array}\right.
$$

where $k_{1}$ and $k_{2}$ are positive gains. The first equation in (9) denotes the virtual control law for the evolution of the virtual target moving along the path, which introduces an extra degree of freedom in the whole path following control design.

Replacing (9) into (8), it leads to

$\dot{V}_{1}=-k_{1} x_{e}{ }^{2}+y_{e} v_{t} \sin \psi_{l o s}-k_{2}\left(\psi_{e}-\psi_{l o s}\right)^{2}$.

With the heading reference designed in (6), there is

$\dot{V}_{1}=-k_{1} x_{e}{ }^{2}-\frac{v_{t} y_{e}^{2}}{\sqrt{y_{e}^{2}+\Delta^{2}}}-k_{2}\left(\psi_{e}-\psi_{l o s}\right)^{2}$.

That means $\dot{V}_{1}<0$ anywhere except the origin, if the AUV travels with a permanent positive surge speed.

\subsubsection{Dynamics controller}

In the overall control loop, the kinematic controller acts as a reference subsystem, giving the desired signal to the control subsystem based on the dynamics level. Using backstepping techniques $[26,27]$, the control law in the kinematic level can be extended to deal with AUV dynamics.

Let $r_{d}$ (desired yaw rate) be the reference signal of $r$ (actual yaw rate), and assume the desired surge speed of the AUV is known as $u_{d}$. Note that the error dynamics between the desired orientation of the AUV and the LOS guidance is $\dot{\psi}_{e}=r_{d}+\dot{\beta}-c_{c} \dot{s}$. By resorting to $\dot{\psi}_{e}$ in (9), the yaw rate reference can be given as:

$r_{d}=\dot{\psi}_{l o s}-y_{e} v_{t} \frac{\sin \psi_{e}-\sin \psi_{l o s}}{\psi_{e}-\psi_{l o s}}-k_{2}\left(\psi_{e}-\psi_{l o s}\right)-\dot{\beta}+c_{c} \dot{s}$.

By applying the backstepping technique, the difference between the actual angular/surge speed and the desired one can be reduced to zero. Consider the candidate Lyapunov function $V_{2}$ :

$V_{2}=V_{1}+\frac{1}{2}\left[\left(u-u_{d}\right)^{2}+\left(r-r_{d}\right)^{2}\right]$.

In order to make the derivative of the Lyapunov function $V_{2}$ negative, we choose

$$
\left\{\begin{array}{l}
\dot{r}=\dot{r}_{d}-k_{3}\left(r-r_{d}\right)-\left(\psi_{e}-\psi_{\text {los }}\right) \\
\dot{u}=\dot{u}_{d}-k_{4}\left(u-u_{d}\right) .
\end{array}\right.
$$

Recalling the error dynamics $\dot{x}_{e}, \dot{y}_{e}$ and $\dot{\psi}_{e}$ in (5), $\dot{s}=k_{1} x_{e}+$ $v_{t} \cos \psi_{e}$ in the kinematic control law (9), and using the desired yaw rate in (12), the derivative of $V_{2}$ can be written as

$$
\begin{aligned}
\dot{V}_{2}= & -k_{1} x_{e}^{2}-k_{2}\left(\psi_{e}-\psi_{\text {los }}\right)^{2} \\
& -y_{e}^{2} \frac{v_{t}}{\sqrt{y_{e}^{2}+\Delta^{2}}}-k_{3}\left(r-r_{d}\right)^{2}-k_{4}\left(u-u_{d}\right)^{2} .
\end{aligned}
$$

It concludes that $\dot{V}_{2}<0$ anywhere except the origin.

Consequently, by combining (9) and (14), the control laws of virtual input $\dot{s}$, the input force and toque $\tau_{u}, \tau_{r}$ are given as:

$$
\left\{\begin{array}{l}
\dot{s}=k_{1} x_{e}+v_{t} \cos \psi_{e} \\
\tau_{u}=m_{u}\left(\dot{u}_{d}-k_{4}\left(u-u_{d}\right)\right)-m_{v} v r+d_{u} u \\
\tau_{r}=m_{r}\left(\dot{r}_{d}-k_{3}\left(r-r_{d}\right)-\left(\psi_{e}-\psi_{l o s}\right)\right)-m_{u v} u v+d_{r} r
\end{array}\right.
$$

where $\tau_{v}=0$ due to the underactuation in the sway direction.

Proposition 1 (Path Following : Under-actuated AUV). Consider an under-actuated AUV with dynamics equations in (1) and (2). Assume the surge speed $u \geq u_{\min }>0$ and the LOS guidance is denoted in (6). Let control laws be given by (16) for some $k_{i}>0(i=1,2,3,4)$, the equilibrium point $\left(x_{e}, y_{e}, \psi_{e}\right)=0^{3}$ is uniformly globally asymptotically and locally exponentially stable (UGASEULES).

Proof. The Lyapunov function $V_{2}$ given by (13) is positive definite and radially unbounded. The derivative of Lyapunov function $\dot{V}_{2}$ given by (15) is negative definite since $v_{t} \geq u \geq u_{\min }>0$. Hence, by standard Lyapunov arguments, $x_{e}, y_{e},\left(r-r_{d}\right)$, and $\left(\psi_{e}-\right.$ $\left.\psi_{\text {los }}\right)$ uniformly global asymptotically converge to 0 . Recalling (6), $\psi_{\text {los }}$ converges to $y_{e}$, and $y_{e}$ converges to 0 subsequently, such that $\psi_{e}$ has the same characteristics with $y_{e}$ and uniformly global asymptotically converges to 0 in the end.

For $\left|y_{e}\right| \leq \bar{y}_{e}$, there is $\frac{v_{t}}{\sqrt{y_{e}^{2}+\Delta^{2}}} \geq \frac{v_{t \min }}{\sqrt{\bar{y}_{e}^{2}+\Delta^{2}}}>0$. By choosing $\min \left\{k_{1}, k_{2}, k_{3}, \frac{v_{\text {tmin }}}{\sqrt{\bar{y}_{e}^{2}+\Delta^{2}}}\right\}=k_{\min }$, the derivative of Lyapunov function (15) becomes

$$
\begin{aligned}
\dot{V}_{2} \leq & -k_{\min }\left[x_{e}^{2}+y_{e}^{2}+\left(\psi_{e}-\psi_{\text {los }}\right)^{2}\right. \\
& \left.+\left(r-r_{d}\right)^{2}+\left(u-u_{d}\right)^{2}\right] \leq-2 k_{\min } V_{2} .
\end{aligned}
$$

It means the derivative of the candidate Lyapunov function is quadratically negative definite. Hence, the equilibrium point $\left(x_{e}, y_{e}, \psi_{e}\right)=0^{3}$ is uniformly locally exponentially stable (ULES) with the convergent rate of $2 k_{\min }$, and the region of ULES depends on $\Delta$. 


\subsubsection{Evolution of side-slip angle}

Clearly, the evolution of side-slip angle $\ddot{\beta}$ is requested for control computation of torque input $\tau_{r}$ in (16) as $\dot{r}_{d}=f(\ddot{\beta})$ can be derived from (12), but the second derivative of side-slip angle cannot be directly measured in practice. Moreover, $\beta$ is not directly controlled for under-actuated AUV and cannot converge to a desired side-slip angle rigorously, as there is no lateral actuator contributing force to steer the sway speed $v$ for an under-actuated AUV traveling in the horizontal plane.

By adopting the similar method in [28], one can resort to the original dynamic model of the AUV for the computation of $\ddot{\beta}$. There is

$\ddot{\beta}=\frac{1}{v_{t}^{2}}(u \ddot{v}-v \ddot{u})-2 \frac{v_{t}^{2}}{v_{t}} \dot{\beta}$.

The accelerations of speed can be estimated by

$\left\{\begin{array}{l}\ddot{u}=\ddot{u}_{d}+k_{4}^{2}\left(u-u_{d}\right) \\ \ddot{v}=-\left(m_{u} \dot{u} r+m_{u} u \dot{r}+\dot{d}_{v} v+d_{v} \dot{v}\right) / m_{v} .\end{array}\right.$

The angular acceleration implicitly used in (17) is gained through non-trivial algebraic computation

$\dot{r}=f_{r d} /\left(1-\frac{m_{u}}{m_{v}} \cos ^{2} \beta\right)$

where

$$
\begin{aligned}
f_{r d}= & \ddot{\psi}_{l o s}+c_{c} \ddot{s}+\frac{\partial c_{c}}{\partial s} \dot{s}^{2} \\
& -k_{1}\left(\dot{\psi}_{e}-\dot{\psi}_{l o s}\right)-\left(\psi_{e}-\psi_{l o s}\right)-k_{3}\left(r-r_{d}\right) \\
& +\frac{\ddot{u} v}{v_{t}^{2}}+2 \frac{\dot{v}_{t}}{v_{t}} \dot{\beta}+\frac{u}{v_{t}^{2}}\left(\frac{m_{u} \dot{u} r+\dot{d}_{v} v+d_{v} \dot{v}}{m_{v}}\right) .
\end{aligned}
$$

It is noted that $\dot{r}$ is causal and well defined in (18) if

$\frac{m_{u}}{m_{v}}=\frac{m-X_{\dot{u}}}{m-Y_{\dot{v}}}<1$.

As the added mass $Y_{\dot{v}}$ is negative in a real fluid [20], the condition (20) holds if an AUV is stern dominant [29], i.e., $-Y_{\dot{v}}>-X_{\dot{u}}>0$.

\subsection{Path following control design for fully-actuated AUV}

The kinematic equations in (1) are in the same form for both the fully-actuated vehicle and under-actuated vehicle. Using the fact that

$\left\{\begin{array}{l}u=v_{t} \cos \beta \\ v=v_{t} \sin \beta .\end{array}\right.$

Replacing above equations into (2), the dynamics of fully-actuated AUV can be rewritten in terms of $\left(v_{t}, \beta, r\right)$ as

$$
\left\{\begin{array}{l}
\dot{v}_{t}=f_{v t}\left(v_{t}, \beta, r\right)+\tau_{v t}\left(\tau_{u}, \tau_{v}, v_{t}, \beta\right) \\
\dot{\beta}=f_{\beta}\left(v_{t}, \beta, r\right)+\tau_{\beta}\left(\tau_{u}, \tau_{v}, v_{t}, \beta\right) \\
\dot{r}=f_{r}\left(v_{t}, \beta, r\right)+\tau_{r} / m_{r}
\end{array}\right.
$$

where

$$
\left\{\begin{aligned}
f_{v t}= & \left(\frac{m_{v}}{m_{u}}-\frac{m_{u}}{m_{v}}\right) v_{t} r \sin \beta \cos \beta \\
& -\left(\frac{d_{u}}{m_{u}} \cos ^{2} \beta+\frac{d_{v}}{m_{v}} \sin ^{2} \beta\right) v_{t} \\
f_{\beta}= & -\left(\frac{m_{v}}{m_{u}} r \sin ^{2} \beta+\frac{m_{u}}{m_{v}} r \cos ^{2} \beta\right) \\
& +\left(\frac{d_{u}}{m_{u}}-\frac{d_{v}}{m_{v}}\right) \sin \beta \cos \beta \\
f_{r}= & -\frac{d_{r}}{m_{r}}+\frac{m_{u r}}{m_{r}} v_{t}^{2} \sin \beta \cos \beta
\end{aligned}\right.
$$

with

$$
\left\{\begin{array}{l}
\tau_{u}=\left(m_{u} \cos \beta\right) \tau_{v t}-\left(m_{u} v_{t} \sin \beta\right) \tau_{\beta} \\
\tau_{v}=\left(m_{v} \sin \beta\right) \tau_{v_{t}}+\left(m_{v} v_{t} \cos \beta\right) \tau_{\beta} .
\end{array}\right.
$$

The transformation between $\left(\tau_{v t}, \tau_{\beta}\right)$ and $\left(\tau_{u}, \tau_{v}\right)$ is nonsingular due to the determinate of the transformation $m_{u} m_{v} v_{t} \neq 0$.

As the side-slip angle $\beta$ is directly controlled by the control input $\tau_{v}$ in the fully-actuated configuration, we can guarantee the desired side-slip angle. This is the main difference from that of under-actuated AUV where the side-slip angle is indirectly controlled. Hence, the desired side-slip angle can be predefined as $\beta_{d}$ in fully-actuated case. By choosing the Lyapunov function $V_{\beta}=\frac{1}{2}\left(\beta-\beta_{d}\right)^{2}$, it renders

$\dot{\beta}=\dot{\beta}_{d}-k_{5}\left(\beta-\beta_{d}\right)$

where the gain $k_{5}>0$.

Therefore, the control input of $\tau_{\beta}$ in (21) is

$\tau_{\beta}=-f_{\beta}+\dot{\beta}_{d}-k_{5}\left(\beta-\beta_{d}\right)$

which drives $\beta$ asymptotically converging to $\beta_{d}$.

The only difference between fully-actuated and under-actuated AUV configurations, exists whether the side-slip angle $\beta$ is directly controlled or not. The common point is that control inputs for $\tau_{u}$ and $\tau_{r}$ are the same for both cases. Directly using the same virtual control input $\dot{s}$, surge and yaw control inputs as proposed in (16), and replacing (25) into (23) to get the sway control input $\tau_{v}$, there is

$$
\left\{\begin{array}{l}
\dot{s}=k_{1} x_{e}+v_{t} \cos \psi_{e} \\
\tau_{u}=m_{u}\left(\dot{u}_{d}-k_{4}\left(u-u_{d}\right)\right)-m_{v} v r+d_{u} u \\
\tau_{r}=m_{r}\left(\dot{r}_{d}-k_{3}\left(r-r_{d}\right)-\left(\psi_{e}-\psi_{\text {los }}\right)\right)-m_{u v} u v+d_{r} r \\
\tau_{v}=\frac{m_{v} v_{t}}{\cos \beta}\left[\frac{\sin \beta}{m_{u} v_{t}} \tau_{u}-f_{\beta}+\dot{\beta}_{d}-k_{5}\left(\beta-\beta_{d}\right)\right] .
\end{array}\right.
$$

Note that the control law for $\tau_{v}$ is singular when $\beta=\frac{\pi}{2}+$ $2 k \pi, k \in \mathfrak{R}^{+}$. However, $\beta=\frac{\pi}{2}+2 k \pi$ means that the surge velocity $u=0$ and only sway velocity $v$ exists. Actually, the assumed permanent positive surge speed $u>0$ excludes this singularity problem. Therefore, we can state the following proposition for path following control of fully-actuated AUV.

Proposition 2 (Path Following : Fully-actuated AUV). Consider a fully-actuated AUV with the dynamics equations in (1) and (2). Assume the surge speed $u \geq u_{\min }>0$ and the LOS guidance is denoted in (6). Let control laws be given by (26) for some $k_{i}>$ $0(i=1,2, \ldots, 5)$, the equilibrium point $\left(x_{e}, y_{e}, \psi_{e}\right)=0^{3}$ is uniformly globally asymptotically and locally exponentially stable (UGASEULES).

The proof is similar to the under-actuated case and omitted here.

Furthermore, the acceleration of side-slip angle $\ddot{\beta}$ is still required to compute control input $\tau_{r}$, as $\ddot{\beta}$ is implicitly included in $\dot{r}_{d}$ which appears in $\tau_{r}$. Fortunately, it is far more easier to get it compared with that in the under-actuated model. As the $\beta$ is directly controllable in (24), there is

$\ddot{\beta}=\ddot{\beta}_{d}+k_{5}^{2}\left(\beta-\beta_{d}\right)$.

We can conclude that the difference between two path following controllers for under-actuated and fully-actuated AUV is that, the side-slip angle is directly controlled in fully-actuated vehicle due to the available control input $\tau_{v}$, so we can use (27) to replace (17) in the under-actuated AUV controller. The control inputs for $\tau_{u}$ and $\tau_{r}$ are the same for both of the cases.

From this point of view, we can keep the control framework to be consistent for both under-actuated and fully-actuated configurations, as we just need to switch the control computation for $\ddot{\beta}$ between (17) and (27). However, the smooth control transition is preferred to the hard switch in practical case. 


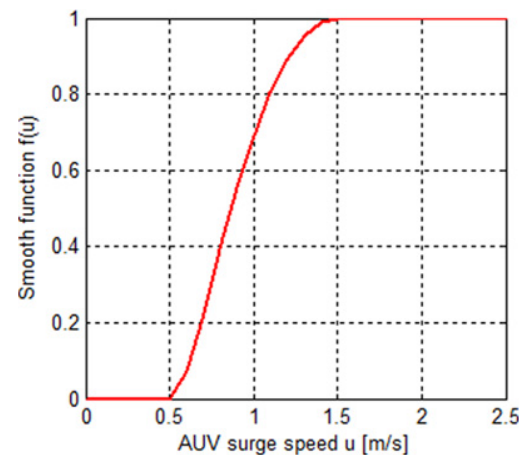

(a) Slacking factor $k_{u}=1.0$

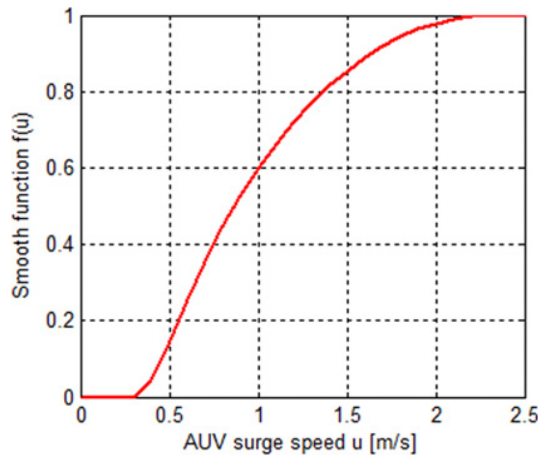

(b) Slacking factor $k_{u}=0.5$.

Fig. 3. Smooth function for AUV configuration transition.

\subsection{Smooth transition from fully-actuated to under-actuated AUV configuration}

While an AUV moves from low-speed at beginning to highspeed traveling, the forward speed is the key factor that makes an AUV with tunnel thrusters in the lateral direction to behave like an under-actuated or a fully-actuated configuration. Basically, when the forward movement of an AUV is in high-speed mode, the efficiency of the lateral actuator is dramatically decreased due to the relative perpendicular water flow passing the outlet, such that a fully-actuated AUV degenerates into an under-actuated one. Therefore, an individual AUV inherently experiences both fully-actuated and under-actuated configurations during single voyage, and the design of smooth continuous transition from fullyactuated to under-actuated configuration is indeed necessary. In this paper, the smooth transition factor is proposed to directly relied on the forward surge speed of an AUV, such that the control design for $\tau_{u}$ and $\tau_{r}$ in both fully-actuated and under-actuated cases can be the same as shown in (16) and (26), and the only consideration for smooth transition goes into the design of control effort in the sway direction.

Given the surge speed of an AUV as

$0<u_{\min } \leq u_{1}<u_{2} \leq u_{\max }$

where the bounded value $u_{\min }$ is the lower threshold of the surge speed $u$ when the AUV is still fully-actuated, and $u_{\max }$ is the upper threshold of $u$ when the AUV becomes under-actuated.

In order to achieve a smooth continuous transition from fullyactuated to under-actuated AUV configuration, a natural choice is constructing the transition factor $f(u)$ as a function of the instantaneous surge speed $u$, that is

$f(u)=\frac{1}{2}\left\{1+\sin \left[\frac{\pi}{2} \operatorname{sat}\left(k_{u} \frac{u^{2}-u_{1} u_{2}}{u\left(u_{2}-u_{1}\right)}\right)\right]\right\}$

where $k_{u}>0$ is a slack variable, and $\operatorname{sat}(\cdot)$ is a saturation function in $[-1,1]$ such that $0 \leq f(u) \leq 1$. Note some sigmoid functions, for instance, $\tanh (\cdot), \operatorname{atan}(\cdot)$, are excluded to build the transition factor as only $0<f(u)<1$ is guaranteed.

In Fig. 3(a) and (b), the smooth function in (29) for fullyactuated and under-actuated AUV transition is illustrated with $u_{1}=0.5 \mathrm{~m} / \mathrm{s}, u_{2}=1.5 \mathrm{~m} / \mathrm{s}$, and the slacking factor $k_{u}$ is set as 1.0 and 0.5 , respectively.

Revisiting the control law (26) for the fully-actuated case and using the fact of null sway force in the under-actuated case, the control input in the sway direction can be written as

$\tau_{v}=\left\{\begin{array}{c}\tau_{v 1}=0, \quad \text { underactuation } \\ \tau_{v 2}=\frac{m_{v} v_{t}}{\cos \beta}\left[\frac{\sin \beta}{m_{u} v_{t}} \tau_{u}-f_{\beta}+\dot{\beta}_{d}-k_{5}\left(\beta-\beta_{d}\right)\right], \\ \text { fully actuation. }\end{array}\right.$
Table 1

Parameters of AUV dynamics model.

$\begin{array}{lll}m=185 \mathrm{~kg} & I_{z}=50 \mathrm{~kg} \mathrm{~m}^{2} & \\ X_{u}=-70 \mathrm{~kg} / \mathrm{s} & Y_{v}=-100 \mathrm{~kg} / \mathrm{s} & N_{r}=-50 \mathrm{~kg} \mathrm{~m}^{2} / \mathrm{s} \\ X_{\dot{u}}=-30 \mathrm{~kg} & Y_{\dot{v}}=-80 \mathrm{~kg} & N_{\dot{r}}=-30 \mathrm{~kg} \mathrm{~m}^{2} \\ X_{u|u|}=-100 \mathrm{~kg} / \mathrm{m} & Y_{v|v|}=-200 \mathrm{~kg} / \mathrm{m} & N_{r|r|}=-100 \mathrm{~kg} \mathrm{~m}^{2}\end{array}$

The smooth transition of the control force in the sway direction, from low-speed to high-speed maneuvering, can be proposed as

$\tau_{v}=f(u) \tau_{v 1}+(1-f(u)) \tau_{v 2}$

where $f(u)$ is given in (29) and $\tau_{v 1}, \tau_{v 2}$ are given in (30). When the AUV maneuvers in high-speed approaching to $u_{2}$, it tends to $\tau_{v}=\tau_{v 1}$ (i.e., underactuation) due to the dramatically decreased efficiency of its lateral actuator in high-speed forward movement. It tends to $\tau_{v}=\tau_{v 2}$ (i.e., fully actuation) vice versa.

Hence, we can get the proposition for path following control of AUV maneuvering in the full-range speed profile (from low-speed to high-speed) as follows:

Proposition 3 (Path Following : Smooth Transition From Fullyactuated to Under-actuated AUV Configuration). Consider an AUV with the kinematic equations in (1), and the unified dynamics Eq. (2) for both the under-actuated and fully-actuated AUV configurations. Assume the surge speed $u \geq u_{\min }>0$ and the LOS guidance is denoted in (6). The control inputs of $\dot{s}, \tau_{u}, \tau_{r}$ are given in (26). Let the control law for control force $\tau_{v}$ in the sway direction be given by (31). Then, a smooth continuous transition between low-speed and highspeed path following control can be achieved.

The proof can be made by combining the Propositions 1 and 2 .

\section{Numerical simulations}

In order to illustrate the performance of the proposed control scheme in Section 3, numerical simulations are carried out with the AUV dynamics model in $[30,16]$. The hydrodynamic parameters are shown in Table 1.

\subsection{Path parameterization}

Suppose that the AUV in the mission is required to follow an ' $S$ '-shape path given in Cartesian coordinates, which is parameterized as

$x_{s}(\eta)=\sum_{i=1}^{5} a_{i} \eta^{i-1}, \quad y_{s}(\eta)=\sum_{i=1}^{5} b_{i} \eta^{i-1}$

where the path coefficients are given in Table 2. 
Table 2

Parameters of desired path.

\begin{tabular}{llllll}
\hline Coefficients/index & 1 & 2 & 3 & 4 & 5 \\
\hline$a_{i}$ & 0. & 0.87 & -0.02 & $-10^{-6}$ & $1.5 \times 10^{-6}$ \\
$b_{i}$ & 0. & 0.5 & $-10^{-3}$ & $-10^{-5}$ & $10^{-7}$ \\
\hline
\end{tabular}

Table 3

Control parameters of AUV path following.

\begin{tabular}{llll}
\hline$k_{0}=1.0$ & $k_{1}=0.1$ & $k_{2}=10$ & $k_{3}=1.0$ \\
& $k_{4}=0.1$ & $k_{5}=1.0$ & $k_{u}=1.0$
\end{tabular}

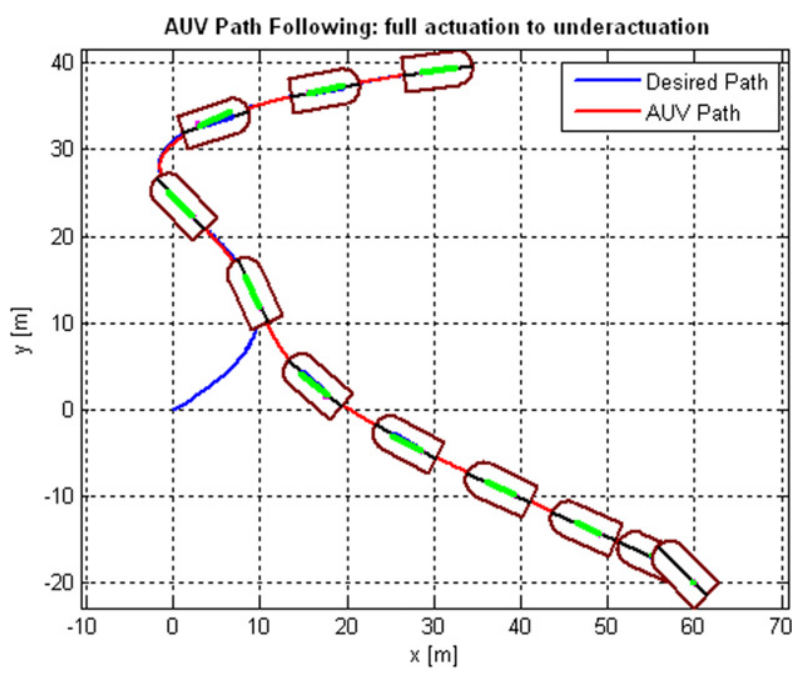

Fig. 4. Spatial convergence of AUV path following.

The desired path is parameterized by along path distance $s$. The evolution of $s$ is constrained by the virtual control law in (16), such that $s(t)$ can be computed. Hence, $s$ is known in the simulation while a precise estimation of the function $\eta(s)$ is unknown. However, it can be achieved by integration of

$\frac{d \eta}{d s}=\frac{1}{\sqrt{x_{s}^{\eta}(\eta)^{2}+y_{s}^{\eta}(\eta)^{2}}}$

where $x_{s}^{\eta}(\eta)=\frac{\partial x_{s}(\eta)}{\partial \eta}$ and $y_{s}^{\eta}(\eta)=\frac{\partial y_{s}(\eta)}{\partial \eta}$. The heading of the virtual target in the inertial frame is $\psi_{F}(s)=\arctan \frac{y_{s}^{\eta}(\eta)}{x_{s}^{\eta}(\eta)}$, and the path curvature is $c_{c}(s)=\frac{\partial \psi_{F}(s)}{\partial \eta} \frac{d \eta}{d s}$ at the virtual target's position.

\subsection{Analysis of control performance}

In the path following mission, the control objectives are as follows: (1) regulate the distance from the AUV to the virtual target moving on the desired path to zero; (2) regulate the course direction of the composite speed of the AUV to zero relative to the tangential direction of the desired path.

In the numerical simulation, initial conditions of the AUV are set as:

$u(0)=0.1 \mathrm{~m} / \mathrm{s}, \quad v(0)=0 \mathrm{~m} / \mathrm{s}, \quad r(0)=0 \mathrm{rad} / \mathrm{s}$,

$x(0)=60 \mathrm{~m}, \quad y(0)=-20 \mathrm{~m}$,

$\psi(0)=3 \pi / 4 \mathrm{rad}, \quad s(0)=0 \mathrm{~m}$.

The desired surge speed is $u_{d}=2.0 \mathrm{~m} / \mathrm{s}$ with $\dot{u}_{d}=0, \ddot{u}_{d}=0$. It means that the AUV travels through low-speed $(u(0)=0.1 \mathrm{~m} / \mathrm{s})$ and high-speed $\left(u_{d}=2.0 \mathrm{~m} / \mathrm{s}\right)$, such that it experiences both fullyactuated and under-actuated configurations.
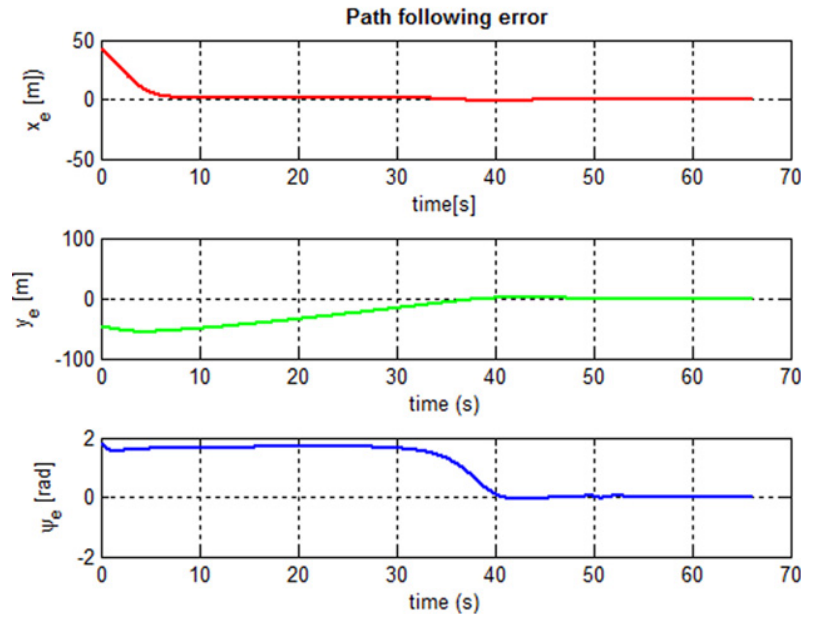

Fig. 5. Relative posture errors between AUV and virtual target.
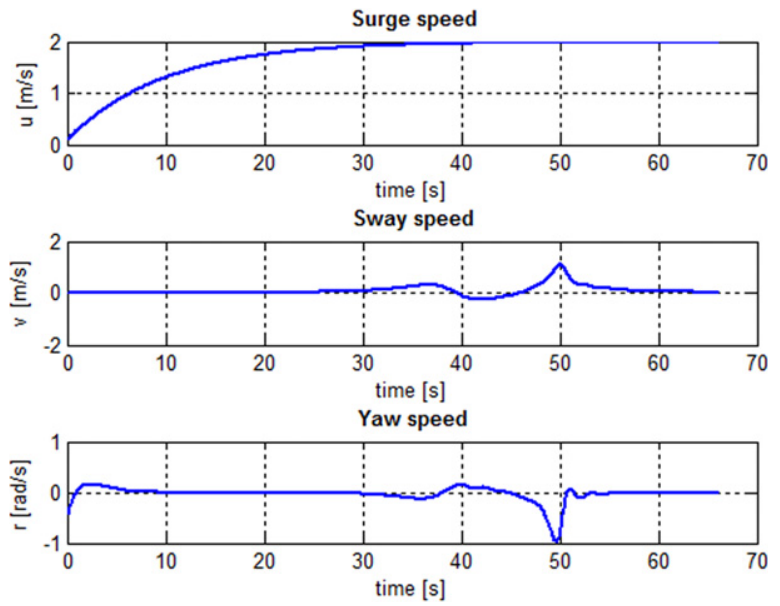

Fig. 6. Velocities profiles of AUV.

The thresholds for transition function in (29) are given as $u_{1}=$ $0.5 \mathrm{~m} / \mathrm{s}, u_{2}=1.0 \mathrm{~m} / \mathrm{s}$, and the control parameters are given in Table 3.

The reference path and the AUV path are shown in Fig. 4, which indicates the AUV follow the path well with the help of the snap shot of AUV movement. The path following errors of AUV, i.e., $x_{e}, y_{e}, \psi_{e}$, are asymptotically converging to zero in Fig. 5 . The velocity profiles of AUV are illustrated in Fig. 6, where the AUV achieves the desired surge speed $u_{d}=2.0 \mathrm{~m} / \mathrm{s}$ in the end.

Note that in Fig. 7 how the virtual target collaboratively adjust its speed (slow-down/wait/speed-up/hold) to help the AUV follow the desired path. This is achieved by introducing an extra degree of freedom to control the virtual target in the path following control design as described in the paper.

In Fig. 8, it clearly shows that the side-slip effect cannot be ignored as the maximum value of $\beta$ is around $0.5 \mathrm{rad}$, and its acceleration also varies during the path following stage. It concludes that the computation effort on the side-slip angle proposed in this paper is valuable.

\subsection{Analysis of transition performance}

In order to show the transition performance from fully-actuated to under-actuated configuration, the AUV is demanded to move with surge speed from $0.1 \mathrm{~m} / \mathrm{s}$ to $2 \mathrm{~m} / \mathrm{s}$, i.e., traveling from lowspeed at beginning to high-speed cruise stage. Moreover, the difference of sway force efforts between the fully-actuated and 

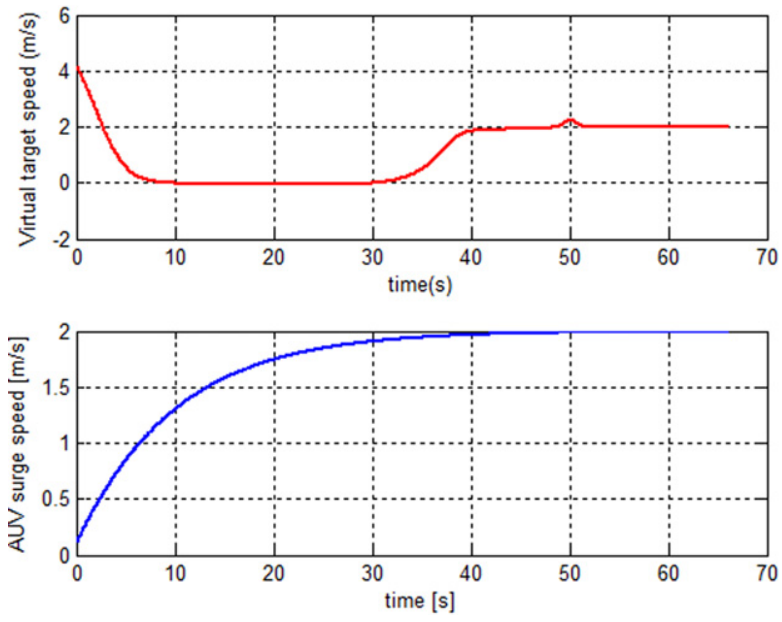

Fig. 7. Speed comparison with virtual target and AUV.
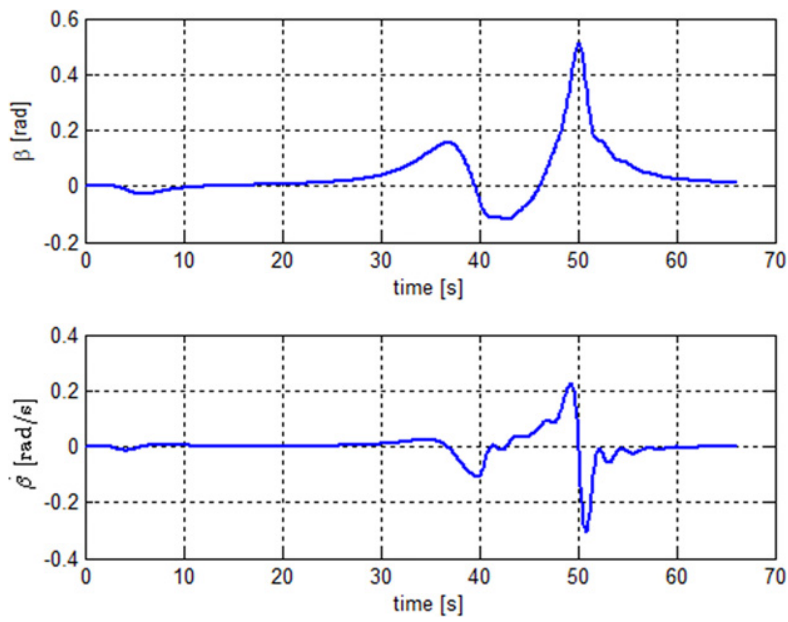

Fig. 8. Computation of side-slip angle.

under-actuated cases is shown by setting different slacking factor of the transition function.

In Figs. 9 and 10, the control efforts of the surge/sway force and yaw torque are clearly illustrated in both fully-actuated and underactuated configurations, where the shadow areas clearly indicate the transition period from fully-actuated (low-speed at beginning) to under-actuated configuration (high-speed traveling) while the AUV following the desired path. The enough time for smooth transition allows the stability between the control switching from fully-actuated to under-actuated AUV configurations [31,32].

By setting the slacking factor $k_{u}=1.0$ in Eq. (29) such that the transition function is illustrated in Fig. 3(a), the smooth transition period from fully-actuated to under-actuated configuration exactly exists between the lower threshold $u_{1}=0.5 \mathrm{~m} / \mathrm{s}$ and the upper threshold $u_{2}=1.5 \mathrm{~m} / \mathrm{s}$, when the AUV surge speed $u$ belongs to $[0.1,2] \mathrm{m} / \mathrm{s}$. Hence, the AUV travels in purely under-actuated case and $\tau_{v}=0$ keeps constantly in Fig. 9 after the transition stage with $t \in[2.4,13.2] \mathrm{s}$, which clearly revealed the underactuated characteristic with no lateral force but only the non-zero surge force $\tau_{u}$ and yaw torque $\tau_{r}$ contributing the control efforts to maneuver through the sharp ' $U$ ' turn in Fig. 4 around time stamp $t=50 \mathrm{~s}$

In order to distinguish different sway control efforts effected by the transition stage, by deliberately setting the slacking factor $k_{u}=0.5$ in Eq. (29) such that the transition function is illustrated in Fig. 3(b), the smooth transition period exists between $u_{1}=$ $0.3 \mathrm{~m} / \mathrm{s}$ and $u_{2}=2.4 \mathrm{~m} / \mathrm{s}$. Since the surge speed $u \in[0.1,2] \mathrm{m} / \mathrm{s}$,
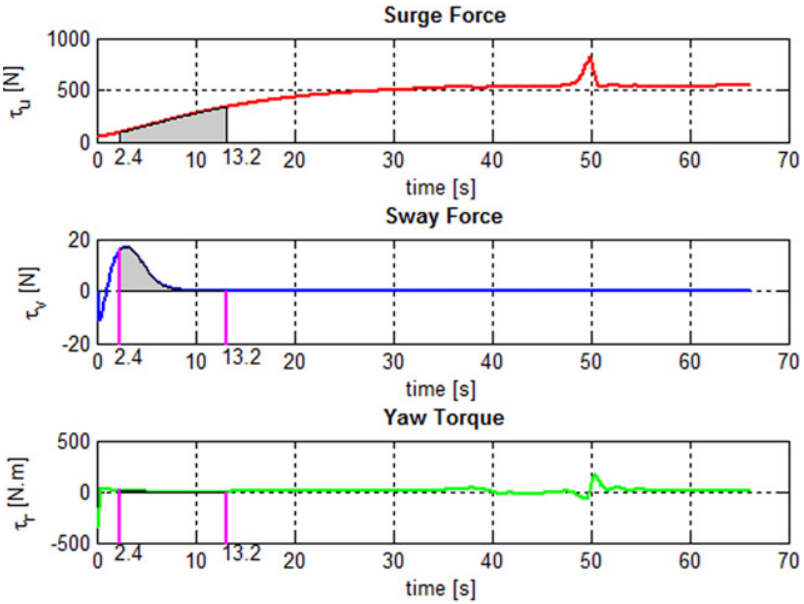

Fig. 9. Transitions of AUV control inputs $\left(k_{u}=1.0\right)$.
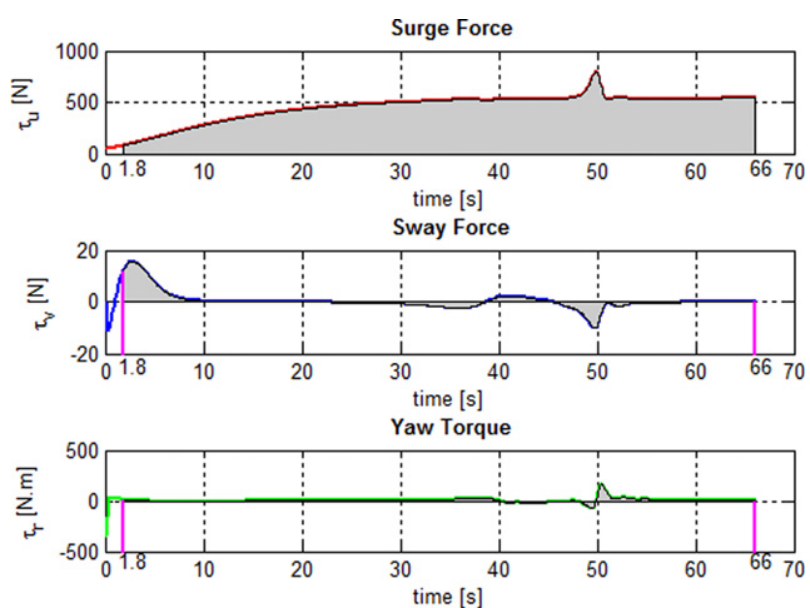

Fig. 10. Transitions of AUV control inputs $\left(k_{u}=0.5\right)$.

the AUV keeps staying in the transition stage from fully-actuated to under-actuated configuration after the purely fully-actuated stage at beginning with $t \in[0,1.8] \mathrm{s}$, which is clearly revealed in Fig. 10 with $\tau_{v} \neq 0$ contributing control effort in the sway direction as well except that $\tau_{u} \neq 0, \tau_{r} \neq 0$ when the AUV travels along the sharp ' $U$ ' turn around time stamp $t=50 \mathrm{~s}$.

\section{Conclusions}

This paper addresses the problems of nonlinear motion control of path following for fully-actuated/under-actuated AUV in the horizontal plane, based on Lyapunov theory and backstepping technique. Traditional LOS guidance for tracking straight-line path is trimmed to follow curved path by building LOS in the moving Frenet-Serret frame. Smooth transition between fullyactuated and under-actuated AUV configurations is achieved in a single synthesized controller, which enables an AUV travels throughout the full-range speed profiles from low-speed to highspeed maneuvering. Finally, the simulation results illustrate the performance of the proposed controllers. Future work will include the solutions to parameter uncertainty and the presence of ocean current.

\section{Acknowledgments}

This work was partially supported by the EU FP6 FreeSubNet project under Grant 036186, the National Natural Science Foundation (NNSF) of China under Grant 51209100, the Specialized Research Fund for the Doctoral Program of Higher Education under 
Grant 20120142120045, and the Fundamental Research Funds for the Central Universities (HUST: 2013TS090). The first author was supported by the European Marie Curie ESR Fellowship of the FP6 project Freesubnet.

The authors would like to thank the anonymous reviewers for their valuable comments and suggestions to improve the quality of this paper.

\section{References}

[1] D. Richard Blidberg, R.M. Turner, S.G. Chappell, Autonomous underwater vehicles: Current activities and research opportunities, Robot. Auton. Syst. 7 (2-3) (1991) 139-150.

[2] J. Yuh, Design and control of autonomous underwater robots: a survey, Auton. Robots 8 (1) (2000) 7-24.

[3] T.B. Curtin, D.M. Crimmins, J. Curcio, M. Benjamin, C. Roper, Autonomous underwater vehicles: trends and transformations, Mar. Technol. Soc. J. 39 (2005) 65-75.

[4] E. Bovio, D. Cecchi, F. Baralli, Autonomous underwater vehicles for scientific and naval operations, Annu. Rev. Control 30 (2) (2006) 117-130.

[5] N. Leonard, Control synthesis and adaptation for an underactuated autonomous underwater vehicle, IEEE J. Ocean. Eng. 20 (3) (1995) 211-220.

[6] K.D. Do, J. Pan, Z.P. Jiang, Robust and adaptive path following for underactuated autonomous underwater vehicles, Ocean Eng. 31 (16) (2004) 1967-1997.

[7] A. Aguiar, J. Hespanha, Trajectory-tracking and path-following of underactuated autonomous vehicles with parametric modeling uncertainty, IEEE Trans. Automat. Control 52 (8) (2007) 1362-1379.

[8] L. Lapierre, Robust diving control of an auv, Ocean Eng. 36 (1) (2009) 92-104.

[9] C. Woolsey, L. Techy, Cross-track control of a slender, underactuated auv using potential shaping, Ocean Eng. 36 (1) (2009) 82-91.

[10] D. Bertin, L. Branca, Operational and design aspects of a precision minewarfare autopilot, in: Proceedings of the Warship 2000, London, UK., 2000.

[11] K. Claes G., Autopilot and track-keeping algorithms for high-speed craft, Control Eng. Pract. 8 (2) (2000) 185-190.

[12] R. Skjetne, S. Moi, T. Fossen, Stabilization of sets parametrized by a single variable: application to ship maneuvering, in: Fifteenth International Symposium on Mathematical Theory of Networks and Systems University of Notre Dame, Vol. 2, August 12-16, 2002, pp. 1699 - 1704

[13] K.D. Do, Z.P. Jiang, J. Pan, H. Nijmeijer, A global output-feedback controller for stabilization and tracking of underactuated odin: a spherical underwater vehicle, Automatica 40 (1) (2004) 117-124.

[14] M. Breivik, T. Fossen, A unified control concept for autonomous underwater vehicles, in: American Control Conference, 2006, Minneapolis, Minnesota, USA., 2006, pp. 4920-4926.

[15] T.I. Fossen, Guidance and Control of Ocean Vehicles, Wiley, New York, 1994.

[16] F. Repoulias, E. Papadopoulos, Planar trajectory planning and tracking control design for underactuated auvs, Ocean Eng. 34 (11-12) (2007) 1650-1667.

[17] L. Lapierre, B. Jouvencel, Robust nonlinear path-following control of an auv, IEEE J. Ocean. Eng. 33 (2) (2008) 89-102.

[18] L. Lapierre, D. Soetanto, A. Pascoal, Nonlinear path following with applications to the control of autonomous underwater vehicles, in: Proceedings of $42 \mathrm{nd}$ IEEE Conference on Decision and Control, Vol. 2, 2003, pp. 1256-1261.

[19] Nomenclature for Treating the Motion of a Submerged Body Through a Fluid, in: Technical and Research Buletin of the Society of Naval Architects and Marine Engineers, SNAME, 1950, pp. 1-5.

[20] T.I. Fossen, Marine Control Systems: Guidance, Navigation and Control of Ships, Rigs and Vehicles, Marine Cybernetics AS, Trondheim, 2002.

[21] R. Hindman, J. Hauser, Maneuver modified trajectory tracking, in: Proc. of Int. Symp. Mathematical Theory Networks and Systems, St. Louis, MO, USA., 1996, pp. 1473-1479.

[22] P. Encarnacao, A. Pacoal, M. Arcak, Path following for autonomous marine craft, in: Proceedings of the 5th IFAC Conference on Manoeuvring and Control of Marine Craft, 2000, pp. 117-122.

[23] A. Micaelli, C. Samson, Trajectory Tracking for Unicycle-type and Twosteering-wheels Mobile Robots, Research Report RR-2097, INRIA, 1993.
[24] K. Pettersen, E. Lefeber, Way-point tracking control of ships, in: Decision and Control, 2001. Proceedings of the 40th IEEE Conference on, Vol. 1, 2001, pp. 940-945.

[25] T. Fossen, M. Breivik, R. Skjetne, Line-of-sight path following of underactuated marine craft, in: Proceedings of the $6^{\text {th }}$ Conference on Manoeuvring and Control of Marine Craft (MCMC 2003), Girona, Spain, 2003.

[26] I.K.M. Krstic, P. Kokotovic, Nonlinear and Adaptive Control Design, Wiley, New York, 1995.

[27] H.K. Khalil, Nonlinear Systems, third ed., Prentice-Hall, Upper Saddle River, NJ, 2002.

[28] L. Lapierre, D. Soetanto, Nonlinear path-following control of an auv, Ocean Eng. $34(11-12)$ (2007) 1734-1744.

[29] E.V. Lewis, Principles of Naval Architecture, Vol. III, second ed., SNAME, Jersey City, NJ, 1989.

[30] K. Pettersen, O. Egeland, Time-varying exponential stabilization of the position and attitude of an underactuated autonomous underwater vehicle, IEEE Trans. Automat. Control 44 (1) (1999) 112-115.

[31] D. Liberzon, A. Morse, Basic problems in stability and design of switched systems, IEEE Control Syst. 19 (5) (1999) 59-70.

[32] A. Aguiar, J.P. Hespanha, A.M. Pascoal, Switched seesaw control for the stabilization of underactuated vehicles, Automatica 43 (12) (2007) 1997-2008.

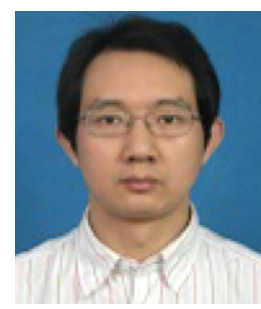

Xianbo Xiang received the Ph.D. degree in robotics from the University of Montpellier 2, Montpellier, France, in 2011. He received his Bachelor and Master degrees in Automatic Control and Marine Engineering, from Huazhong university of science and technology, China, in 2000 and 2003 respectively. And then, he joined the same university as a Lecturer. From Sept. 2006 to Dec. 2006, he was an EU Erasmus Mundus visiting scholar in the SpaceMaster project. From February 2008 to March 2011, he worked for the European Project FreeSubNet as a EC Marie Curie ESR Fellow at LIRMM, CNRS UMR 5506, France. Currently, he is an Associate Professor at the School of Naval Architecture and Ocean Engineering, Huazhong University of Science and Technology.

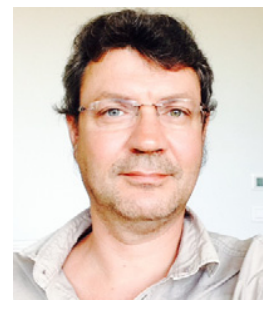

Lionel Lapierre received the Ph.D. degree in robotics from the University of Montpellier 2, Montpellier, France, in 1999. Then, he joined the team of Prof. A. Pascoal within the European project FreeSub for three years in Instituto Superior Técnico(IST), Portugal. Since 2003, he has been with the Robotics Department, the Laboratoire d'Informatique, de Robotique et de Microélectronique de Montpellier (LIRMM), CNRS UMR 5506, Montpellier, France. He is currently a Maître de conférences (eq. Associate Professor) at University of Montpellier 2, Montpellier, France.

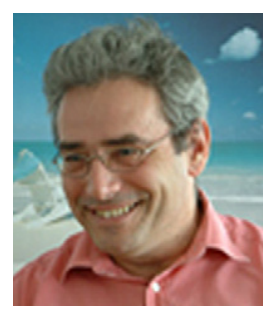

Bruno Jouvencel was born on February 3, 1955, in Paris, France. He graduated from the Electronical Engineering Department, Ecole Normale Suprieure, Cachan, France, in 1981. He received the Ph.D. degree in perception systems for robotic manipulators in automatic control from Montpellier University, Montpellier, France, in 1984 Currently, he is a Full Professor at Montpellier University II and Researcher at LIRMM. For ten years, he has worked on the design, the command, and the perception of autonomous underwater vehicles. A first prototype Taipan 1 has been developed in 1998, and a second vehicle Taipan2(H160) was conceived and realized in partnership with an industrial company. He was recipient of the first prize of the EURON/EUnited Robotics "Technology Transfer Award" in 2006 due to the contribution to Taipan AUVs. 